\title{
“Oops...”: Mobile Message Deletion in Conversation Error and Regret Remediation
}

\author{
MARK WARNER, Northumbria University, UK \\ LAURA LASCAU, University College London, UK \\ ANNA L. COX, University College London, UK \\ DUNCAN P. BRUMBY, University College London, UK \\ ANN BLANDFORD, University College London, UK
}

\begin{abstract}
Message deletion in mobile messaging apps allows people to "unsay" things they have said. This paper explores how and why people use (or do not use) this feature within remediation strategies after a communication error is identified. We present findings from a multi-stage survey designed to explore people's general experiences of the message deletion feature $(N=401)$, peoples' experiences of using this feature during the remediation of an error $(N=70)$, and receivers' perceptions around recent message deletions $(N=$ 68). While people are typically aware of the deletion feature, it is infrequently used. When used, it is primarily done so to improve conversations by reducing confusion between conversation partners. We found people being aware of message deletions creating information-gaps which can provoke curiosity in recipients, causing them to develop narratives to help address the uncertainty. We found concerns amongst senders that these narratives would be of a negative nature, having an undesirable impact on how others perceive them. We use our findings to suggest ways in which mobile messaging apps could improve conversational experiences around erroneous and regrettable messages.
\end{abstract}

CCS Concepts: • Human-centered computing $\rightarrow$ Empirical studies in collaborative and social computing; User studies.

Additional Key Words and Phrases: mobile messaging, messaging apps, message deletion, regrettable messages, global delete, unsent messages, errors in communication, messaging errors, delete notification, delete indicator

ACM Reference Format:

Mark Warner, Laura Lascau, Anna L. Cox, Duncan P. Brumby, and Ann Blandford. 2021. “Oops...”: Mobile Message Deletion in Conversation Error and Regret Remediation. In CHI Conference on Human Factors in Computing Systems (CHI '21), May 8-13, 2021, Yokohama, Japan. ACM, New York, NY, USA, 20 pages. https://doi.org/10.1145/3411764.3445118

\section{INTRODUCTION}

We often fail to say what we intended to say; errors and regrets in communication are common place. When speaking, our words can run ahead of our thoughts and this can lead to linguistic errors and dead ends that are often repaired as we speak. These can range from giving incorrect information (e.g., telling someone the incorrect time for a flight), to slips of the tongue (e.g., when the UK Prime Minister accidentally described the UK election as 'career-defining' when he meant 'country-defining') [4]. People also say things that they later come to regret [24], often after a period of reflection [23]. Writing is an all-together slower and more reflective activity that allows for more planning, yet errors and regrets can still occur. The challenge with many computer-mediated communications (CMC) is that they rely on

Permission to make digital or hard copies of all or part of this work for personal or classroom use is granted without fee provided that copies are not made or distributed for profit or commercial advantage and that copies bear this notice and the full citation on the first page. Copyrights for components of this work owned by others than ACM must be honored. Abstracting with credit is permitted. To copy otherwise, or republish, to post on servers or to redistribute to lists, requires prior specific permission and/or a fee. Request permissions from permissions@acm.org.

(c) 2021 Association for Computing Machinery.

Manuscript submitted to ACM 
text-based communications which are often exchanged quickly (e.g., chat messages rapidly exchanged between online conversation partners). As a result errors and regrets within messages can and do occur [1, 10, 29, 36], from small errors like typos to significant regrets within messages such as oversharing of personal information.

Similarly across both face-to-face (f2f) and CMC, there is the risk that errors and regrets in communication could negatively impact a person's image [32,36]. If a person was to accidentally say something they did not mean, this could lead to an undesirable impression developing around them. To mitigate errors and regrets in f2f communication, people utilise various remediation (or 'repair') strategies as part of their broader impression management strategy [16]. However, text-based CMC are distinctly different from f2f. Words spoken in error are not recorded; they must be recalled from an often less than perfect human memory with verbal corrections and conversation repairs helping to minimise their impact [24]. In contrast, text-based CMC systems typically keep a conversation history, with errors and regrets contained within messages being persistent in these histories. Recognising this, technology designers have implemented deletion functions across many of today's CMC platforms (e.g., mobile messaging apps, social media). Deletion functions allow for sent communications to be deleted after they are sent. In mobile messaging apps (e.g., WhatsApp), an indicator is often provided to indicate that a message was deleted for all parties of the conversation (Figure 1). These indicators alert receiver(s) to the event, but not the content of the deleted message unless it was read prior to deletion.

Existing research has highlighted how people engage in various remediation strategies when errors occur in f2f communications [16, 22, 27], and after regrets are identified in online social networks sites (SNSs) [36]. However, prior studies have not considered (1) mobile messaging apps and their message deletion functions, or (2) the way deletions are perceived by recipients. It is important to consider mobile messaging apps distinct from SNSs as they offer a less networked and more intimate CMC experience. Moreover, as erroneous and regrettable message remediation is an impression management behaviour [16], understanding how message deletions are perceived by receivers is important to understand the effectiveness of these functions. This paper looks to address these gaps by asking the following research question: How and why are message deletions used in the remediation of error and regrets in messages, and how are these perceived by recipients?

To address this research question, we report on a study which used a multi-stage survey method. The initial stage was designed to elicit generalised perceptions around a technology and also acted as a screening tool for follow-up surveys. The next stage allowed us to obtain behavioural insights around a technology by probing both senders and receivers experiences of deletions. In this study, this meant obtaining behavioural insights from single message deletion incidents. In this way, we were able to gain insights from the perspective of senders and recipients of deleted messages in both pairwise (one-to-one) and group (one-to-many) chats.

Our work provides a number of contributions to the HCI and social computing literature. Firstly, we provide empirical insights into why people use (or do not use) the message deletion function as a remedial strategy when communicating using mobile messaging platforms, showing that people can adopt novel strategies in new communication contexts. Secondly, we identify how deletions can result in negative perceptions forming which can have impression management implications on senders of deleted messages. Finally, we discuss the implications of our research and suggest ways in which mobile messaging apps could improve conversational experiences around erroneous and regrettable messages. Our work focuses on predominately mobile-based messaging apps (e.g., WhatsApp, Facebook Messenger), rather than apps that are predominately desktop-based (e.g., Teams, Slack). 


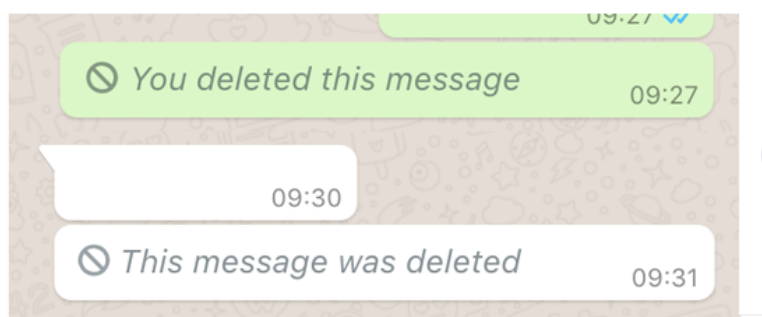

A

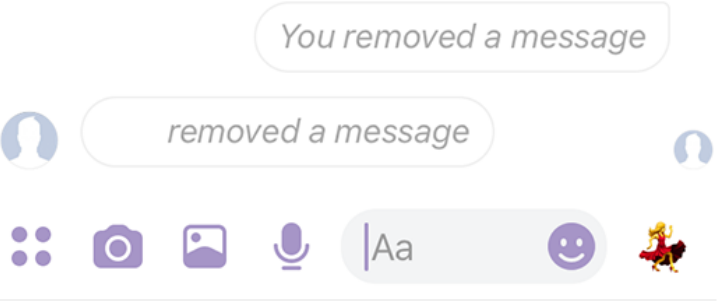

B

Fig. 1. Examples from (A) WhatsApp and (B) Facebook Messenger apps of message having been deleted from both a sender's and recipient's perspective. N.B.: In the case of Facebook Messenger, delete notifications now read 'You unsent a message' instead of 'You removed a message'.

\section{BACKGROUND}

\subsection{Online Communication Errors, Regrets, and Impression Management}

Errors and regrets in online communications can be embarrassing and even harmful as they can result in information being revealed that the sender never intended to reveal [17]. From an impression management perspective [8], this can be problematic as people look to maintain the boundaries between multiple online identities which form their self-concept (e.g., WhatsApp groups for family, friends, and work), and maintain relationship dynamics. Online, the separation of people into groups can support information disclosure, with relationship dynamics being an important factor in information disclosure decisions [14]. Whilst early work suggested a lack of social cues may limit support for relationship dynamics (e.g., maintaining social status) within text-based CMC environments [15], Boucher et al.'s [3] work suggests that interlocutors are equally sensitive to each others thoughts and feelings in text-based CMC as in $\mathrm{f} 2 \mathrm{f}$ communication environments. Yet, positive impressions may still be harder to form in CMC when compared with f2f interactions [25].

Unlike f2f boundary management where people can often delineate boundaries by place and time (e.g., workplace, home time), online communications make boundaries between identities more permeable as they lack physical place and are not separated by time. While people work to manage these boundaries online [41], this increase in permeability and lack of compatibility between different identities can make online information disclosures far more problematic [6]. Providing people with the ability to delete previously disclosed information may support people in managing multiple online identities, and in the process of remediation after a communication error has been identified.

McRoberts et al. [21] used Goffmans's theory of self-presentation [8] to understand people's use of ephemeral messages in Snapchat (i.e., messages that disappear after a brief time period). They found the feature being used to test out content on their audience prior to posting permanently, with the period of ephemerality providing users time to reflect on their post's content. This form of ephemeral messaging may help alleviate concerns around errors in CMC, due to its reduced permanency. Message deletion (explicit or ephemeral) may help to increase the control people have over the dissemination of information and how they are perceived by others (e.g., [29, 36]). However, designing content deletion options in online CMC environments is challenging. Most popular messaging apps that we investigate in this research replace deleted content with a residual delete indicator (e.g., "This message was deleted"). Prior work [5, 37, 39] suggests that where expected information is missing, negative inferences may develop around that missing information, making it more challenging for people to align the impression they are looking to "give" with the one that is "given 
off" [8]. Information gap theory suggests that awareness of missing information can prompt a strong desire to fill in these gaps to increase clarity and reduce uncertainty [9]. Yet, if a negative outcome is anticipated, a person may purposefully avoid seeking information to fill the gap.

\subsection{Errors, Regrets, and Remediation Strategies}

We are imperfect creatures: it is what makes us human. We make errors when communicating and interacting, and these can sometimes be sources of regret. In this section, we explore research on communication errors, regrets, and remedial strategies in both f2f and CMC.

Knapp et al. [16] highlight different types of regrettable messages in f2f communication that includes less obvious blunders, such as asking how someone's relative is without knowing they are recently deceased, to deceptive messages, messages containing direct or implied criticisms, and those that revealed too much information. When exploring errors and regrettable messages in $\mathrm{CMC}$, the majority of research is focused on online social networking platforms, with Facebook and Twitter receiving the most attention. Wang et al. [36], in their study of regrettable messages on Facebook, found similarities to findings in f2f communication, as well as new forms, such as posts sent to unintended audiences, users underestimating the consequences of their posts, misunderstandings around the way the platform works, and usability problems. Regrettable experiences that arise in Facebook because of the user's own actions have also been found to increase rates of abandonment of the platform [10].

When Twitter accounts were observed, around half of all users deleted at least one sent tweet [1], with a substantial number of these being deleted for superficial reasons such as correcting typos, a finding supported by other research [12]. Sleeper et al. [29] found Twitter users to be more likely to regret posts that were overly expressive, or that revealed too much personal information which may in part be due to privacy concerns [40]. Zhou et al. [42] explored prediction of regrettable tweets, applying content based features to a set of deleted tweets; they found that tweets containing negative sentiment, those relating to relationships, or containing curse words or references to sex were the most likely to be considered regrettable and deleted.

While there has been a persistent interest from the HCI community in information deletion more generally (e.g., [11], as well as deletions and regrettable posts in online social networking environments, far less is known about communication errors, regrets, and message deletions in non-networked online social environments such as mobile messaging apps. The one study that has explored message deletions from a usable security standpoint reports various self-reported reasons people engage in message deletion, with improving messages (e.g., correcting typos) being the most frequently reported. The ease with which online apps and platforms allow people to communicate can increase the risk of these types of errors and regrets within messages occurring (e.g. accidentally sending the wrong message to someone in a chat app [13]). The often large networks of contacts that reside within our communication channels (e.g. Facebook social network, large group chats) can result in regrettable messages having a far greater negative impact, with greater levels of audience exposure [29]. This makes understanding remedial strategies and technologies that support such strategies an important focus of research.

When a failure event occurs in f2f communication, research shows people engage in various remediation strategies in an attempt to recover. Scott and Lyman [28] suggest that people account for their actions through excuses where they admit the illegitimacy of their action while limiting their responsibility, or justifications where they admit their responsibility but limit recognition of the event as illegitimate. Schönbach [27] identify two additional strategies: concessions, which is the admission of responsibility either in full or partially by the sender, and refusals, which is the full or partial denial of the event or the deflection of blame by the sender onto others. Expanding on this work, Metts and Cupach [22] 
found people engaging in simple apologies such as "I'm sorry", excuses, justifications, humour, escape, avoidance, which is the lack of recognition of the failure or the remedial strategy of "silence" [20], and aggression. In CMC environments such as Facebook, Wang et al. [36] identified users establishing rules around posting, delaying posts, limiting social network size, self-censoring content, deleting posts, apologising, limiting active engagement, using more select communication channels, engaging with privacy settings, using multiple accounts to understand what others can see on their profile, using fake names or statuses, and listening to offline advice.

In summary, an examination of related work highlights the need to understand how message deletions are used in error and regrets remediation, and how they are perceived by recipients. Specifically, our work aims to answer the following research question: How and why are message deletions used in the remediation of error and regrets in messages, and how are these perceived by recipients?

\section{METHOD}

This section provides details of the survey method developed to understand both general experiences and specific experiences around recent deletion events, from both the sender's and recipient's perspectives. Beyond understanding people's general experiences, we wanted to understand more about the nature and relationship dynamics of the conversations where deletions had been made or observed. To this end, we designed a multi-stage survey approach. The first survey collected general user insights into message deletion and acted as a participant screening tool. The experience surveys were distributed to a subset of the general survey participants and were used to gain insights into specific message delete incidents that participants had recently experienced.

\subsection{Participant Sampling}

Participants were recruited via the academic participant recruitment platform Prolific ${ }^{1}$. This allowed us to compensate participants for their time (2019/20 UK living wage of $£ 8.21$ per hour) and quickly distribute follow-up surveys with selected participants. The inclusion criteria for the general survey was: (1) over the age of 18, and (2) must use at least one mobile phone chat app. A total of $N=401$ participants responded to the general survey call. Participants were evenly balanced in terms of gender (52.6\% female and 45\% male), were young (under 24 (21.9\%), 24-34 (47.6\%), mostly White (83.3\%) from Europe (78.1\%) or North America (15.5\%), with an income that the participant believed to be average (40.9\%) or below average (36.4\%) for their age and group. The experience surveys included the additional inclusion criteria of having experienced a deleted message in a chat app within the last seven days. We received 140 responses to the experience surveys; two participants were removed from the analysis as they completed an incorrect survey for the type of message they found (e.g., completed a survey developed for a message sender when they were the recipient). One response to the experience survey was written in Spanish and was translated to English by the second author.

\subsection{General Survey}

Participants were tasked with using their mobile phone to find a message that had been deleted within the past seven days, and asked if they would like to participate in a follow-up survey. All participants were then asked questions related to the message delete feature (surveys included as supplementary material).

\footnotetext{
$\overline{{ }^{1} \text { https://www.prolific.ac }}$
} 


\subsection{Experience Surveys}

We developed four experience surveys:

(1) Recipient_Pairwise $(N=33)$ - someone else deleted a message in a pairwise chat.

(2) Recipient_Group $(N=35)$ - someone else deleted a message in a group chat.

(3) Sender_Pairwise $(N=35)$ - the participant deleted a message in a pairwise chat.

(4) Sender_Group $(N=35)$ - the participant deleted a message in a group chat.

The day after distributing the general survey, all participants who reported having found a recently deleted message were sent an experience survey. Participants were asked to locate and answer questions related to the message they had found the previous day (surveys included as supplementary material). To limit the amount of participant work and effort and to gain a broader range of experiences, participants reported on only a single message delete experience. A recent experience was used to reduce the risk of misreporting due to poor memory recall.

\subsection{Data Analysis}

Collected data consisted of structured (e.g., selection boxes) and unstructured (e.g., free text) response fields. All data was analysed by the first and second authors. The structured data was analysed descriptively using SPSS, reporting frequencies of responses.

Data collected from unstructured fields (e.g., free text) was analysed collaboratively with the authors initially reading through the data to increase their familiarly and discuss initial insights. A part inductive, part deductive approach to analysis of this data was used. Using NVivo 12, an initial round of inductive coding was performed by the two authors, with one coder independently coding a column of responses to create a codebook by applying descriptive codes to the text. Where no code was applied, it was marked and later discussed between the first and second authors. The developed codes were collaboratively discussed, with similar codes being collapsed, and coding names and descriptions being refined.

Inter-rater reliability was used across the coded data as the authors intended to report the results both qualitatively and quantitatively and wanted a measure of consistency [19]. The Cohen's (unweighted) kappa $\kappa$ was calculated across all coded columns, with a minimum of $\kappa=0.73$ reported, showing a good degree of inter-rater reliability. After discussing disagreements, the codes developed for each column were again collaboratively discussed with similar codes being collapsed. This codebook was then used to deductively code the same data. Some responses were coded with more of a specific focus than others due to the more direct nature of the question being asked. For instance, responses to the question "What reason did they give for deleting the message?" focused on coding the types of reasons participants described for deletion.

As the purpose of this study was to generate qualitative insights supported by descriptive statistics, we did not design the surveys in such a way that would allow us to perform any form of significance testing. For example, in order to understand whether a message had been read prior to deletion, the four experience surveys asked slightly different questions with different response scales, depending on whether the message was deleted in a pairwise or group conversation, and whether the participants were recipients or senders of the deleted message. Therefore, we do not report the statistical significance of our results. 


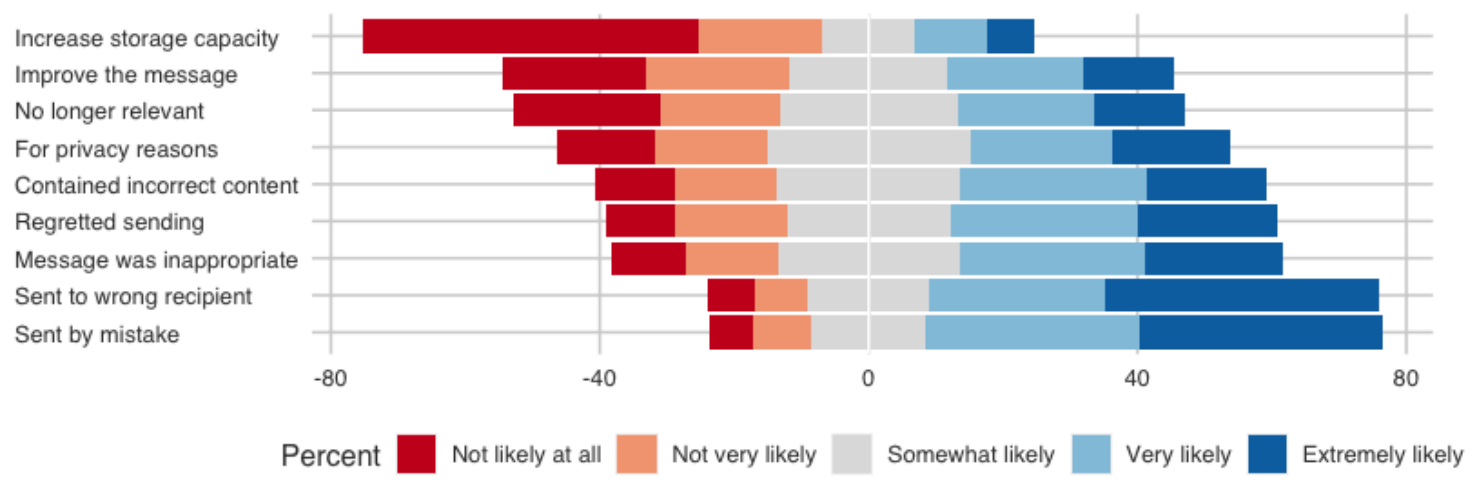

Fig. 2. Overview of most likely reasons reported for deleting messages shown in a diverging stacked bar chart. Positive responses are stacked to the right of a vertical baseline and negative responses are stacked to the left of this baseline. Mid-point responses ("Somewhat likely") are evenly split across positive and negative stacks.

\subsection{Ethical Considerations}

We considered the ethics of conducting this study through internal discussions during the study development and in writing of institutional ethics review board application. Through these deliberations, no significant concerns were raised; however, we were mindful that participants could disclose sensitive information through the unstructured fields in the surveys which could expose them to certain risks. We regularly prompted participants to avoid disclosing anything sensitive, such as names or addresses. Institutional ethics review board approval was obtained with the reference: UCLIC/1819/012.

\section{GENERAL SURVEY FINDINGS}

The two most commonly reported chat apps used by our participants were Facebook Messenger (78.3\%) and WhatsApp $(73.8 \%)$, with over $96 \%$ of participants reporting use of at least one of these apps. Both of these apps support message deletion, and both leave a residual delete indicator when a message is deleted. Despite WeChat's large user base of over 1 billion monthly active users [30], it was one of the least commonly reported apps used by the participants in our sample (1.5\%). This discrepancy is likely to be due to the demographics of our sampled participants, with low representation of people from Asia (2\%), where this app is primarily used.

\subsection{Frequency and Reasons for Deletion}

From our participants, $61.2 \%$ (245) reported having deleted or seen someone else delete a message within the last seven days. More generally, the majority of the participants $(94.5 \%, 379)$ were aware that they could delete a message after it had been sent. However, they indicated low use of the function, with $75.1 \%$ (301) indicating never or only rarely deleting messages, and $22 \%$ (89) stating that they only sometimes deleted messages.

Most participants $(82.5 \%, 331)$ reported to have seen others delete messages at some point in the past, of which 314 (95\%) reported seeing a delete indicator. Over 77\% (311) reported rarely or only sometimes observing other people delete messages in a chat app that they used. A smaller number of participants $(17.5 \%, 70)$ reported never having seen another person delete a message. 


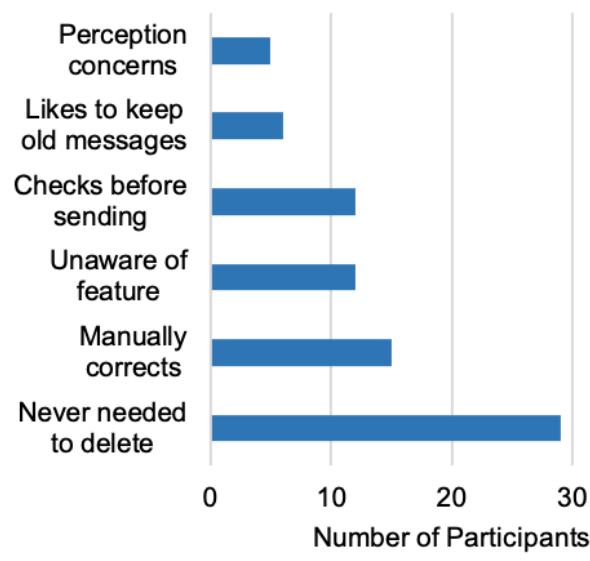

Fig. 3. Reasons participants reported for never using the delete function.

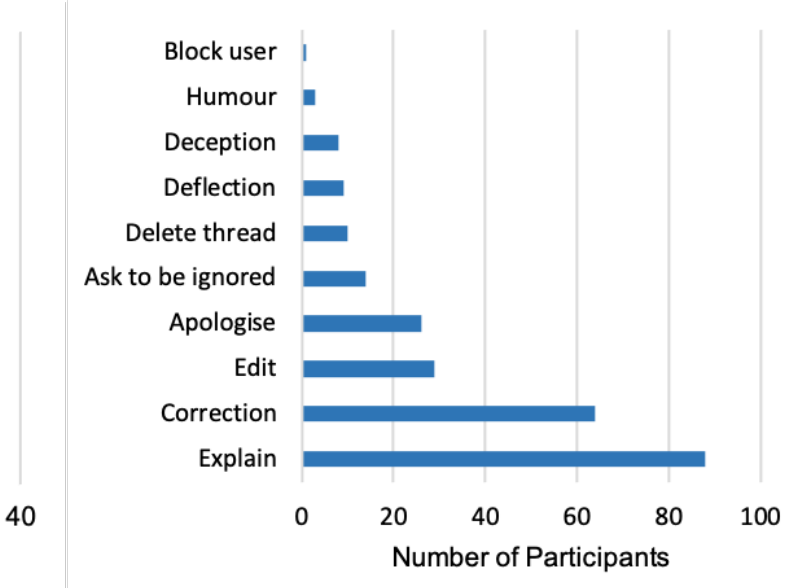

Fig. 4. What participants reported doing instead of using the delete function.

We asked participants how likely they were to delete messages, providing them with a predefined set of reasons based on prior research [26], the results of which are presented in Figure 2. The two most likely reasons for deleting messages were if the message was sent by mistake, or was sent to the wrong recipient. Almost $68 \%$ (272) of the participants reported being extremely or very likely to delete a message if it was sent by mistake, and almost 67\% (268) if the message was sent to the wrong recipient. The least likely reported reasons for deleting messages were to increase storage capacity or to improve a message (e.g., correct a typo).

\subsection{Reasons for Never Using the Delete Function}

Participants who reported having never previously deleted a message were asked what their reason was for never using this function. We received 79 free text responses, summarised in Figure 3. The most common response was a lack of reason to delete (36.7\%). For instance, p251 stated: "II] never had a reason to, [I never] say anything that I need to hide". Others stated a preference for manually correcting messages rather than deleting them (18.9\%), such as using a follow-up message containing a fixed typo often followed by an asterisks $\left(^{*}\right)$. In contrast, others reported rigorously checking messages before sending to avoid having to delete messages (15.19\%).

4.2.1 Alternatives to Deleting. All participants were asked whether there was anything else they would do instead of deleting a message. We received a total of 253 free text responses to this question which were coded and summarised in Figure 4. The most common alternative behaviour was to send a follow-up message (34.8\%) explaining the mistake that had been made. For example, p160 said they would "follow it up with an, 'oops....' message, explaining what I had actually meant, or if it was the wrong recipient, I would say so. Even if I found it a bit embarrassing, I would just laugh it off and style it out.". Others described sending follow-up messages containing corrections (25.3\%), apologies (10.3\%), or instructions to ignore a message (5.5\%). Follow-up corrective messages were reported to correct the entire message or part of the message with the use of an asterisks $\left({ }^{*}\right)$. For example, p26 said that "if it is a simple typo I would maybe type that word again with a star $\left({ }^{*}\right)$ instead of retyping the whole message.". If an edit message function was available, some participants $(11.5 \%)$ reported a preference for using this function instead of deleting. 


\subsection{The Impact of Delete Indicators}

4.3.1 Why delete indicators may stop people deleting. From our participants, $28.4 \%$ (114) reported that delete indicators may stop them from deleting a message. Participants were asked why this was in a free text question. We received 99 responses, which were coded and summarised in Figure 5. The most commonly reported reason was a concern that recipients may develop negative assumptions around their act of deleting. Participants often described how people may assume they had "something to hide" (49.5\%). Reflecting this, p264 stated: "People know I [have] deleted something, so they'll assume it was something bad." Some participants stated a preference for being able to delete without a notification. p262 wanted to avoid negative perceptions forming: "I would prefer people not to know I had deleted the message as it may appear rude", while p269 wanted to maintain privacy and avoid negative impressions forming: "It would stop me because it would potentially draw more attention to a message that I don't want to be noticed. It would also maybe make other people think I said something rude or shady".

Others reported delete indicator (or "notification" as referred to by the following participant) creating awkward or uncomfortable social situations. For example, p228 stated: "In the past, when my girlfriend has seen this notification, she has gotten anxious about why I have deleted something. Similarly, at least 2 friends of mine have thought the same". Here, a number of participants reported an increased hesitation at deleting messages in more emotionally charged conversations. For instance, p238 stated: "if it would be something I sent during an argument [..] I would rather leave it in [.. than delete it in case it looks like I typed something rash.”. This example highlights how the nature of the conversation can influence the development of negative perceptions which we explore in more detail in section 5.2.

4.3.2 Reasons a delete indicator would not stop people from deleting a message. From our participants, 71.6\% (287) reported that the delete indicator would not stop them from deleting a message. We asked these participants why this was, and 185 provided a reason in a free text response. These responses were coded and are summarised in Figure 6 .

The most commonly reported reason for using the delete function, despite the presence of a delete indicator, were that the indicators were lower cost than the cost of the recipient reading the contents of the regrettable message (42.5\%). For instance p33 stated: "If I felt the need to delete a message then I would have reached a point where I care more about people not seeing the content of said message, rather than being worried about people knowing I deleted a message".

Some respondents (31.9\%) saw no reason to be concerned about the indicator as they expected recipients to mostly ignore it. For instance, p62 stated that: "The other person might see the indicator ask why/what I deleted, which is fine. I wouldn't care if a message coming to me was deleted either, I wouldn't even ask about it at all honestly. I like to think most people would mind their own business too.”. Others highlighted how delete indicators increased transparency. For example, p215 stated: "I agree on the policy to show a message was deleted to avoid confusing situations and people declaring they never wrote anything or some situation like that".

While the delete indicator would not stop some participants from deleting a message, some supported deletions with either a follow-up explanatory message (9.73\%), or by providing an explanation if they were asked about the deleted message (3.78\%). Nearly $7 \%$ of respondents reported using some form of deception in follow-up messages to avoid having to reveal the true nature of why they had deleted the message. For example, p219 stated: "you can just say it was a typo/sent on the wrong page and since they can't see it they won't know if that's not true.". 


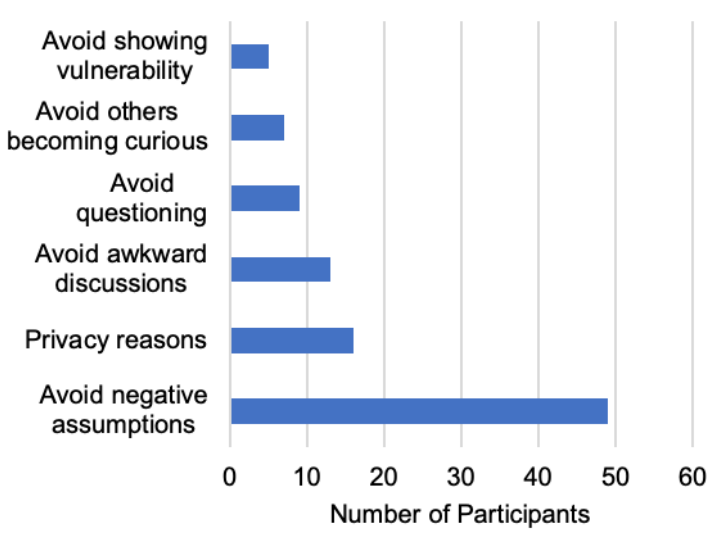

Fig. 5. Reasons participants reported for why delete indicators might stop them from using the deletion function.

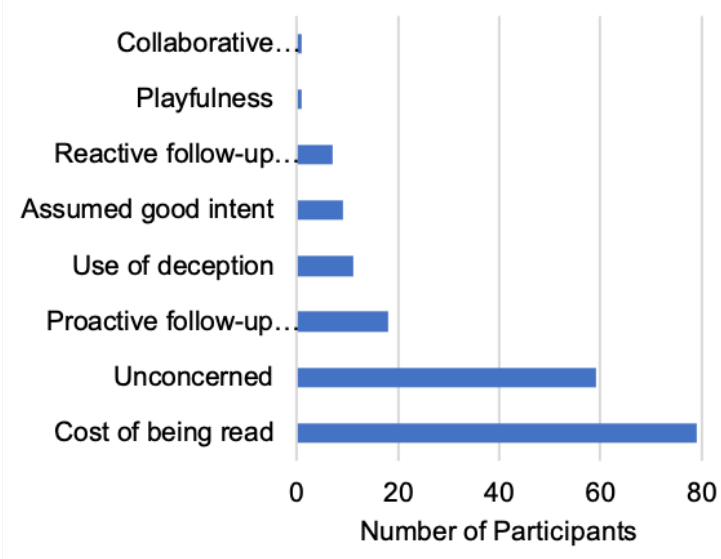

Fig. 6. Reasons participants reported for why delete indicators would not stop them from using the deletion function.

\section{EXPERIENCE SURVEY FINDINGS}

A total of 138 participants completed an experience survey. At the start of each experience survey, participants were asked which mobile chat app they used to send or receive the delete message. The majority of participants reported using either WhatsApp $(60.9 \%, 84)$ or Facebook Messenger $(27.54 \%, 38)$.

\subsection{Sender and Recipient Behaviours}

The most frequently reported reason senders stated for deleting their message (summary in Figure 8) was to remove incorrect content (18.5\%), remove irrelevant information (15.7\%), or improve the message (15.7\%). Of all the senders (pairwise and group) that deleted a message, $27.1 \%$, (19) reported providing a follow-up message to the deletion. In a free-text question, participants were asked the describe the follow-up message they sent. These primarily consisted of justifications or reasons for the deletion, for example, p101 stated "Previous info was wrong so I deleted it sending you now the correct one", while p387 sent an explanation and apology: "I immediately sent a message explaining that $i$ had sent it by mistake and that it was meant for someone else [..] and after that $i$ apologized".

5.1.1 Senders' concerns prior to message deletion. Senders were asked whether they were concerned about how their conversation partner(s) may perceive the deleted message. Most were unconcerned prior to deleting their message $(72.9 \%, 51)$. More senders in pairwise chats were concerned $(37.1 \%, 13)$, compared to those in group chats $(17.1 \%, 6)$. Senders reporting concern were asked to provide detail of their concerns, and we received 19 free text responses. Supporting the general perception findings, the most frequently reported concern was that the act of deleting would be perceived negatively. For example, p362 stated: "I worried they would think I had put something awful [..] and worried they would then judge me on this decision". There was a concern that deletion would signal information being purposefully hidden, and that this would be curiosity provoking, p228 stated "They may wonder why I have deleted the message, did I say something inappropriate or was I hiding something from them”. Others were concerned that message deletion could harm the conversation or the relationship, or simply felt bad for deleting irrespective of how their conversation partner 
may feel. p387 stated: "I didn't exactly have a main concern about how my conversation partner would perceive me deleting the message, but without being able to explain the why, i just felt really bad about [it]. And i immediately sent a message apologizing for my mistake”.

Those unconcerned senders felt that the reason they were deleting was clear, or that the recipient would be able to accurately assume their reason. p214 stated: "As some people just delete messages to correct typos and so on [they would] probably assume it was something to do with that". When one or more chat participants had read the message prior to it being deleted, there was less concern over communicating the reason for deletion. For instance, p187 stated: "for those who saw it, it was absolutely obvious it was a mistake". For others, relationship trust allowed users to delete messages, confidence that no negative assumptions would develop. Moreover, the knowledge that their chat partner(s) could ask them about the message would alleviated any concerns they had prior to deletion.

5.1.2 Receivers' reactions to seeing delete indicator. We asked recipients what their initial reaction was to seeing the delete indicator, and we received 68 free text responses. The most frequent reaction reported was a negative emotional one, with recipients feelings surprised, confused, suspicious, hurt, or experiencing mild fear that they were missing out on something. For instance, p274 stated: "I was a bit curious what was the content. Then I thought maybe the messages were about setting a meeting without me (between the other two of the group) and didn't want me to know about it (a bit suspicious, jealous even maybe-feeling left out?)".

For many recipients, their initial reaction was to develop their own narrative around the sender's reason to delete, with these narratives being used to bridge the uncertainty that deletions can cause. Recipients also reported varying levels of curiosity around what the message may have said and why it was deleted. Some recipients also reported feeling happy that the message was deleted, often as they had read the content and felt that deleting was the most appropriate thing for the sender to do. Finally, a number of recipients found the delete humorous due to the circumstances around the delete or the way in which the delete function was used by the sender, for instance p227 stated: "The deleted message appeared to be sent [in] error because the sender was inebriated and became mixed up about what pictures she had and hadn't sent us. I was amused".

5.1.3 Assumptions that receivers developed around deletions. All 68 of the participants who received a deleted message were asked what they assumed was the reason for the message being deleted, a summary of which is provided in Figure 7. Only $4.4 \%$ (3) of recipients reported developing no assumptions. The majority assumed that messages had been sent to the wrong recipient $(20.6 \%, 14)$, the content was incorrect $(17.7 \%, 12)$, the sender regretted sending the message $(16.2 \%, 11)$, or that it was sent by mistake $(14.7 \%, 10)$.

Our descriptive statistics suggest some variation between pairwise and group chats, with the most frequently assumed reasons in group chats being that the content was incorrect, or the sender regretted sending the message, while messages being sent to the wrong recipient was by far the most frequent assumption reported in pairwise chats. Two recipients selected 'other' as assumed reasons which both related to humour. To give an example, p129 provided a transcript of their conversation where we see the delete function being used as a humorous conversational tool, allowing users to communicate their change of mind with one another through the playful use of message deletes:

person 1: do you guys want to go out tonight?

person 2: yes sure

[p129]: no, im [sic] not [in] the mood today.

person 3: lets [sic] go

person 1: ok, let me change the message. (deletes first message) 


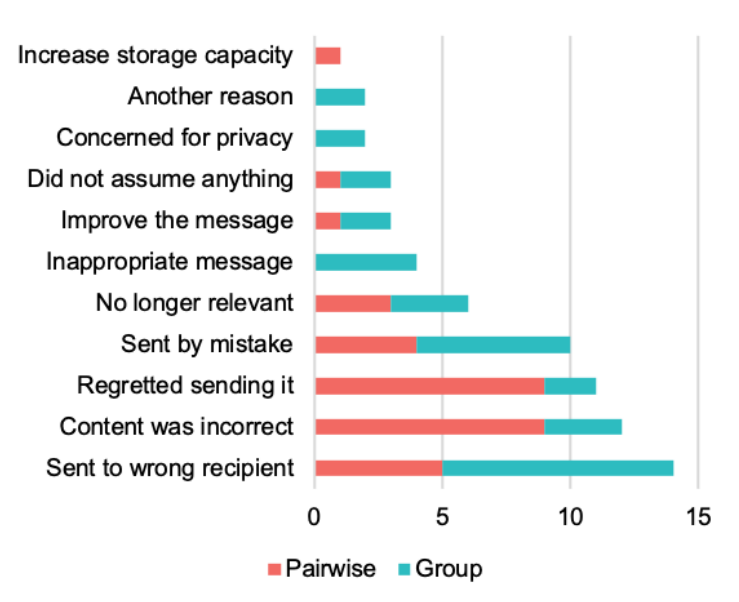

Fig. 7. Recipient's assumed reason for the message being deleted.

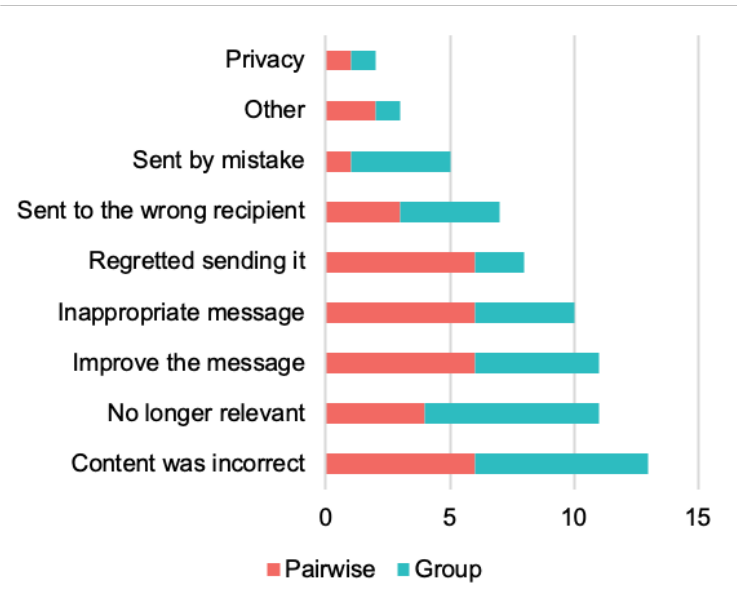

Fig. 8. Senders' reported reason for deleting their message.

person 1: do you guys want to go [..] to a karaoke bar tonight?

[p129]: (deletes first message) YES!

person 1: yea thats [sic] what I thought

Most recipients developed their assumptions from conversational norms and prior behaviours within chats, or by projecting their own behaviours onto the actions of others. In group chats, several recipients reported the speed of conversations being used to develop their assumptions. For example, p179 reported being in a chat with around 50 people where conversation and topic changes happened quickly. As such, she assumed the content of the message was no longer relevant to the topic that was currently being discussed and was deleted to avoid confusion. Similarly, p211 mentioned the speed of the group chat conversation he was part of as his reason for assuming that a message has been deleted, projecting his own prior behaviour onto the actions of another to develop this assumption. The assumption he developed was that the conversation speed caused the sender to type more rapidly to avoid their message being delayed and becoming irrelevant within the context of the conversation, resulting in an error. He stated: "the nature of the chat group means you have to type quickly \& therefore it is easy to make a mistake".

In pairwise chats, we found prior behaviours of chat partners being used to develop assumptions. For instance, p52 received a delete indicator in a chat with her husband and assumed he had sent her a message that was intended for someone else by drawing on her husband's prior behaviour, stating: "That is generally the only reason that $m y$ husband would delete a message based on previous times that this has happened". In the case of p198, her assumption developed based on prior conversational behaviour patterns. She had previously observed her chat partner writing longer messages, and deleting them if typos were present. As a consequence, she would not ask him about the deleted message. She stated: "Well, he tends to write few long messages, instead of many more, but shorter. Due to this habit, he often makes a typo, but he deletes the entire paragraph and starts again. [...] When talking to this particular guy, I have no doubt about the reason for deleting the message." What we see here, and in other behaviours within our data, is people utilising prior behaviours to develop their own narratives to explain the reason behind messages being deleted without directly asking the other party for information or clarification. For instance, p136 thought his conversation partner sent 
either the wrong video, meme or joke while p143 developed a scenarios to explain a message that had been deleted, in which the sender deleted the message to avoid upset of other conversation partners.

Recipients in both pairwise and group chats projected their own behaviours onto others to help them develop narratives around reasons for deletions. p142 developed a narrative that his chat partner had deleted their message to improve it, as this is something he himself does. He stated: "Because I am doing [the] same things a lot. I am very often making mistakes in messages and I am deleting them to write them again.” Similarly, p185 used his own messaging behaviour to develop a narrative around his conversation partner's deleted message, stating: "I would assume the same sort of innocent reasons that I would delete a message, there would be no reason to assume any malice or inappropriateness"

Recipients of deleted messages also used projection as a reason not to delete message. For example, p83 avoided the use of the delete function as he himself had felt uncomfortable in the past when others had deleted messages "I know first person how frustrated [sic] it is to see someone else has deleted a messages be it for a stupid reason or not. Therefore, unless it's something I really don't want other to see I'll refrain from using it just to avoid making the other members uncomfortable.". In the general survey, p11 reported feeling uncomfortable knowing that other people deleted messages in chats, and so avoided deleting messages himself, stating: "because it looks weird and I feel uncomfortable when I see that someone deleted a message so I don't want to do that".

5.1.4 Curiosity around deleted messages. Recipients were asked if they read the message before it was deleted. Most reported having not read the message $(73.5 \%, 50)$. This supports what senders reported, who stated that they mostly deleted messages in less than a minute $(64.3 \%, 45)$ or within 1 to 5 minutes $(22.9 \%, 16)$. Most senders in pairwise chats reported deleting their message before it had been read $(82.7 \%, 29)$, while in group chats most reported nobody $(37.14 \%)$ or only some chat participants (42.9\%) reading their message prior to deletion. Of those, $44 \%(22)$ were either very curious, or extremely curious about what the message originally said, while $46 \%$ (23) were either not at all curious or only somewhat curious. In terms of recipients' curiosity over why the message has been deleted, this was weighted towards the lower end of the scale, with $52.9 \%$ (35) reporting to be either not at all or only somewhat curious, while $47.8 \%$ (32) reported being curious to extremely curious. Most recipients $(85.3 \%, 58)$ reported not asking why a message was deleted, and most reported that the person who deleted the message did not explain their reason for doing so $(75 \%$, 51). Of those that did receive a reason, almost all believed the reason they were given.

The one recipient who did not believe the reason they were provided was in a pairwise chat having an argument with the person they were in a relationship with. The participant reported asking their chat partner "what was that last message?", to which their partner stated it contained a spelling error. However, the participant stated that: "They don't usually care about spelling / grammar errors in messages and wouldn't normally delete messages.". As such, the recipient stated that they "assumed the message was something mean or that would have come across badly". Like the way recipients developed assumptions, here the recipient used the past behaviours of their chat partner, as well as the nature of the conversation, to evaluate the reliability of the reason provided.

Most recipients who did not ask about the deleted message were simply not motivated to do so, or did not apply much importance to the deleted message. Others knew the reason or felt the intention for the delete was obvious, reducing their need to ask. Others felt that asking would be inappropriate, recognising and respecting the privacy of their chat partners, for instance p401 stated: "I think it's a bit impertinent to ask that. If a person wants you to know why they deleted something then they can let you know." Finally, p220 who received a deleted message in a group chat reported not asking because she did not feel she could trust the response from the sender, and stated: "The author would never tell the truth, and I'd rather ask the other members of the group what they think of the deleted message". 


\subsection{Deletions across conversation contexts}

At the start of each experience survey, participants were asked about relationships with chat participants and the nature of the conversation at the time of the deleted message. Across different relationship types, messages were deleted in a variety of conversations. The most frequently reported conversation related to some form of organisation amongst chat partners (30.6\%). For example, organising to meet up, or arranging an event. Around $28 \%$ of conversations were of as general nature with no specific conversation topic. These were described, for example, as "very loose conversation", "general talk", and "routine chit chat" and also including humorous content (e.g., sharing memes). The remaining conversations related to hobbies, enquiries, relationships, work, argumentative, political and socialising. Over half of respondents reported a deleted message incident from a chat with friends (55.8\%), whilst $20.3 \%$ were from family chats, $10.1 \%$ from romantic chats, and the remaining from chats with acquaintance, interest groups (e.g., hobbies), and between professionals. Next, we present insights into message deletion behaviours from a number of conversation contexts.

General and humorous nature. Deleted messages in more general conversations were often low-cost in nature (e.g., typos). For example, p40 was in a "discusion [sic] about the movie" and "made a mistake in spelling", and they used the delete function to remove the error and resend a corrected version of their message. General conversations of a more humorous nature (e.g., people sharing funny videos and memes) were often higher-cost, involving people deleting messages where they perceived them to be upsetting or offensive. For example, p366 sent a "funny meme that had some offensive words" and on reflection, deleted it due to the implicit rule of only using clean language within the chat, and then later "posted the same meme, but editied [sic] with clean language". Another message containing a video was deleted as it depicted a child "doing something stupid" and on reflection was deemed funny, but inappropriate. Whilst messages in general chats were often low-cost errors such as typos, the more humorous of these messages were often misjudged. The initial humorous nature of the content was reflected on and discussed by the poster and other chat participants in a form of collaborative decision-making around what is funny, appropriate, and acceptable within the chat context.

Organisational nature. Deletions within these types of conversations were generally quite pragmatic, deleting to avoid unnecessary confusion or to avoid repetition. For example, p256 stated: "We were trying to arrange dates for a trip", and subsequently described the deleted message as "a previous message referring to other dates than the ones we finally decided", and suggested that "it was better to delete them to avoid confusion." However, deletions were not limited to these types of errors, with a number of other participants reporting to having "side channel" communications with individuals in a group chat when trying to organise something, and mistakenly sending messages to the wrong chat. For instance, p399 stated: "the message I sent was intended to be a private message to someone in the group regarding what we should do, I accidentally sent it to the entire group which could have hurt some feelings if I didn't immediately delete it".

Romantic nature. Conversations of an intimate and emotive nature were mostly between family and romantic contacts. The negative cost associated with messages sent within conversations of this nature were generally perceived to be higher, with the relationships often at stake. For example, p103 stated "We were talking about our parents and I wrote something negative about my mother, as soon I realized it, I deleted the message for the both of us". The participant described using the delete function to "fix" the situation. A number of the conversations where also very emotive in nature, appearing to induce oversharing followed by regret. For example, p25 stated "In the heat $i$ expressed some emotions over a heartbreak $i$ 've been going through. As soon as $i$ hit the send button i realised $i$ told a bit too much so $i$ deleted it." 
Argumentative nature. We have previously highlighted in our general perceptions data analysis how emotionally charged conversations can result in an increased hesitation to delete messages due to concerns around how these deletions may be perceived. Our experience data supports this findings as we see participants engaged in argumentative conversations developing negative assumptions around deletions. For instance, p363 stated having "Argument / disagreement over spending time apart", and further stated that because of the nature of the conversation they "assumed the message was something mean or that would have come across badly". We also see evidence of people reflecting on messages sent during heated exchanges, and the delete feature being used to manage sent regrettable messages. p181 described the relationship of their interlocutor as 'hostile' and stated that "We've spilt up and it was an argument regarding belongings", and that the message "wasn't required, as I was rising to his abuse [..] deleting was better than them seeing what the message said".

\section{DISCUSSION}

This study investigated how and why message deletions are used in the remediation of error and regrets in messages, and how these are perceived by recipients. While people know they can delete messages in mobile messaging apps, we found that people rarely use this feature. When deletions do occur, they are typically to improve conversations to avoid confusion (e.g., delete incorrect information). On the side of the receiver, we find deletions leaving an information-gap, creating uncertainty over why a message was deleted. We also found some recipients developing their own narratives to address this uncertainty. In this section, we discuss the contribution that our findings make beyond the previous research in this area, and also discuss how our findings can be applied to inspire future mobile messaging app designs that better support communication error and regret remediation.

\subsection{Improving communication and reducing confusion.}

We find that people are aware of the deletion feature; however, it is infrequently used. Where it is used, the reason for deletion is different from reasons previously observed across SNSs. Unlike Facebook [36] and Twitter [1, 29, 42], where users tend to delete posts that have an uncertain or negative impact on their online self-presentation, or risk their privacy or safety, we find mobile messaging app users mostly avoid using the delete feature as this can itself result in an uncertain or negative impact on self-presentation. Message deletion is primarily used to "fix" conversations in less emotionally charged conversations. These conversation "fixes" primarily occur to improve the communication and reduce communication uncertainty between chat participants. For instance, we find people deleting incorrect (e.g., wrong movie times) or out of date information (e.g., details of a meeting that is no longer happening) to avoid confusion in the communication.

\subsection{Bridging narratives to fill the information-gap}

As a result of delete notifications (see Figure 1), we find message deletions are themselves a form of communication. We explicitly observed this in the way one participant and his friends deliberately exploited the feature to create a humorous dialogue. However, this feature caused some to be concerned at the negative assumptions that recipients may develop when receiving this notification and the impact this may have on the impression "given off" by the sender, a finding seen in other online environments (e.g., dating [37, 38]). This concern resulted in some users reporting non-use of the feature to avoid the potential negative inferences that may shape around deletions, and the impact this may have on impression management. Yet, for others, the cost of the receiver seeing the message out-weighed the cost of any negative assumptions that the sender may develop. 
As the notification lacks any form of narrative that explains the reason for deletion, it can result in receivers developing what we term "bridging narratives". These narratives are developed to "bridge" the information-gap that is created through these delete notifications. Often, these narratives are developed to reduce the perceived significance of the deleted communication (e.g. explain away the deletion), and to prevent concern growing over the nature of what had been deleted while avoiding direct questioning of the sender. We found people developing these narratives using conversational cues (e.g., conversation speed), which we know from prior work people use to manage their own and evaluate others' self-presentation [5] and in online deception detection [33, 34]. Moreover, we also found people projecting their own past observations and behaviours onto the deletion actions of others, to further support the narratives that they developed around deletions.

\subsection{Non-delete remediation}

Where message deletion is not used, we find people using similar remediation strategies to those used in f2f communication. These include the use of descriptive explanations and apologise as observed previously in f2f communication remediation [22]. What we also see in mobile messaging apps is the use of corrections of content, similar to that found in everyday speech where people regularly repair conversations as they speak [2]. However, the text-based nature of mobile messaging increases the permanency of these errors in communication, resulting in users appropriating technologies to find solutions, such as the use of asterisks $\left(^{*}\right)$ to correct prior erroneous messages.

\subsection{Message deletion in CMC conversation error and regret remediation}

Prior work on remediation of errors and regrets in f2f communications highlight various strategies that people use to manage these types of communication failure events. In this section, we discuss the strategies our research has identified that have been shaped around message deletion.

6.4.1 Avoidance strategy. Where message deletion was used we often found it supplementing existing remediation strategies [20, 22, 27, 28]. Primarily, we found support for avoidance strategies where participants reported avoiding the failure event that had occurred [20]. The notable difference here is that in f2f communication, the error or regret within the communication will be received by the recipient, and so the recipient must accept the avoidance strategy being used by the sender. Message deletion removes the need for this implied social contract between sender and receiver where the message is deleted prior to being read, increasing the effectiveness of this strategy.

6.4.2 Providing a supplementary narrative. Our research identified a concern around message deletion creating an information-gap where uncertainty can develop, resulting in negative assumptions forming. To help mitigate this, we found some participants supporting message deletions by incorporating remediation strategies used in f2f communication such as justifications, reasons, excuses and apologies [22, 28]. This strategy of providing additional follow-up information to explain why a message was deleted allows users to take control of the narrative around why it was deleted, and so minimising the risk of recipients forming undesirable or incorrect "bridging narratives" to explain the deletion. In some instances, people reported incorporating some form of deception into these supplementary narratives. This allows people to remove the original message, take control over the narrative that is developed, and avoid revealing the true reason for the deletion. 


\subsection{Implications for Design}

Communication is complex; when it is computer-mediated, small changes in the way the mediation platform functions can have subtle but significant implications on communication. Our results suggest that deleted messages reduce transparency and can be problematic for the continuity of the conversation chain. A contextualised approach to designing global message delete functions could alleviate concerns, widen appeal, and promote continuity and transparency in communications. We propose a set of recommendations for the design of delete functions in mobile messaging apps.

6.5.1 Make it quicker for users to provide a reason for deleting a message. Our results suggest that people develop their own narratives around senders' reasons to delete, without directly asking the other party for information or clarification. Bridging narratives enable people to manage the information-gap that deletions create, but can result in miscommunication and impact on impression management. As a result, apps could provide users with predefined or customised reasons for deleting a message. For example, after deleting a message, users could be prompted to select a deletion reason from a list of frequently used reasons. The delete indicator could then be customised based on this section, for example: 'This message was deleted as it is no longer relevant', or 'This message was deleted as it was sent by mistake'. Such a feature would need to consider the time criticality of having to delete a message, and should prompt users to provide a reason only after the message has been confirmed as deleted. However, allowing users to provide a reason for deleting a message may result in people feeling pressured to offer an excuse, and an increased expectation from receivers that an explanation be provided. Therefore, further research is needed to understand the implications of such a feature design.

6.5.2 Allowing users to hide messages. In addition to deleting messages, mobile messaging apps could allow users to hide messages which could otherwise have a disruptive effect on a conversation and result in negative perceptions developing if deleted. Hiding messages is based on the idea that there is potential value in enhancing visibility within communications [35], which may also include enhancing visibility of errors. Like in the case of GitHub, where 'off-topic, outdated, or resolved' comments in a repository can be hidden 'to keep a discussion focused' [7], users of mobile messaging apps could also be allowed to hide their messages. Furthermore, as we have seen that people can treat deleted messages with suspicion in certain contexts, allowing users to hide rather than delete messages could alleviate some of the negative perceptions which can develop. However, whilst hiding messages could help with historical error remediation, further research is needed to investigate whether such a feature would help enhance transparency, and hence maintain conversation continuity.

6.5.3 Automated corrections. Drawing on our findings that show how some participants prefer manually correcting messages rather than deleting them, we suggest that the way in which people have appropriated existing text-based $\mathrm{CMC}$ to correct messages could be formalised and automated. For example, a number of participants reported manually correcting errors in their communications (e.g. using asterisk* to correct a time). While messaging platforms that are predominantly desktop-based allow for message editing (e.g., Slack, Teams), this editing feature is not currently supported in the majority of predominantly mobile-based messaging tools, like WhatsApp and Facebook Messenger. We might speculate that the reasons for this difference is due to the differing user interface constraints and/or the primary context of use (e.g., work vs. social). However, a natural language processing (NLP) system could automatically detect these manual corrections in mobile messaging apps (e.g., 'sorry, I meant to say 1pm instead of 2pm'), and correct the original message, eliminating the need for both deleting the message and navigating through a mobile system's interface to edit the content of a message. These forms of corrective systems could help improve conversation quality 
by alleviating confusion through the correction of incorrect information, or the updating of out-of-date or no longer relevant information. Moreover, this would allow users to maintain existing corrective practices, reduce disruption to conversation flow, and enhance usability of the system. However, the reduced autonomy inherent within providing correction instead of deleting messages could result in further errors, and these automated corrections could provide space for uncertainty. Therefore, further work is needed to understand if, and how, an NLP system could be used for error remediation in text-based CMC.

\subsection{Limitations and Future Work}

Our recruitment method limited our sample to mainly white European/North American participants. As communication behaviours differ across cultures [18], the demographics of our sample should be considered when evaluating these findings. However, mobile messaging apps that support message deletion are in common use in both Europe and North America, with 69 million users in U.S alone using WhatsApp [31]. Further work exploring cultural differences in the use of the global message delete feature could be conducted, which could also incorporate different technologies such as WeChat which is more commonly used in China.

The study method used was based on survey instruments which did not allow the researchers to probe participants to gain more in-depth insights into user perceptions and behaviours. Yet, as limited prior work has focused on how and why message deletions are used in the remediation of error and regrets in messages, and how these are perceived by recipients, our findings provide useful new insights that can be used to inform the development of future studies (e.g., semi-structured interviews). For instance, future work could draw from our findings to further explore differences in behaviours across different platforms (e.g., work-based collaboration tools like Slack and Microsoft Teams, email tools, or forums), in different conversational contexts, and between different relationships.

Finally, whilst our study provides recommendations for the design of delete functions in mobile messaging apps, we did not actually ask participants how they envision interacting with the delete function. Asking this was beyond the scope of the study, but our work may provide useful insights to develop future user-centred design work around the delete function. We suggest avenues for future work in the Implications for Design section.

\section{CONCLUSIONS}

We present findings from a multi-stage survey study exploring how and why message deletions are used in the remediation of error and regrets in messages in mobile messaging apps, and how they are perceived by recipients. Our findings point to senders' intentions to remediate conversations in order to reduce communication uncertainty and negative impressions forming. We highlight how information gaps and confusion can arise as a result of deleted messages, and how recipients can develop "bridging narrative" to fill in the information gaps created. Therefore, we extend previous research by exploring people's perceptions of recently deleted messages. We suggest design considerations for in-app delete functions that promote conversation continuity, while incorporating narratives around deletions to reduce concerns that can otherwise develop.

\section{CONTRIBUTIONS}

Warner and Lascau conceptualised and designed the study, collected and analysed the data and wrote the first draft of the paper. Blandford, Brumby and Cox contributed to revising the paper and provided advice and oversight. All authors participated in the development of the ideas presented in this paper. 


\section{ACKNOWLEDGEMENTS}

This work is partially funded by the EU Horizon 2020 research and innovation program under the Marie SkłodowskaCurie Action ITN grant agreement No 675730, by the University College London (UCL) Department of Computer Science, and by the UK Engineering and Physical Sciences Research Council grants EP/N509577/1 and EP/N027299/1.

\section{REFERENCES}

[1] Hazim Almuhimedi, Shomir Wilson, Bin Liu, Norman Sadeh, and Alessandro Acquisti. 2013. Tweets are forever: a large-scale quantitative analysis of deleted tweets. In Proceedings of the 2013 conference on Computer supported cooperative work. ACM, 897-908.

[2] Hans Rutger Bosker, Anne-France Pinget, Hugo Quené, Ted Sanders, and Nivja H De Jong. 2013. What makes speech sound fluent? The contributions of pauses, speed and repairs. Language Testing 30, 2 (2013), 159-175.

[3] Eliane M Boucher, Jeffrey T Hancock, and Philip J Dunham. 2008. Interpersonal sensitivity in computer-mediated and face-to-face conversations. Media Psychology 11, 2 (2008), 235-258.

[4] Dathan, Matt. 2015. David Cameron makes another gaffe: 'This election is all about my career... sorry, I mean country'. //www.independent.co.uk/news/uk/politics/generalelection/david-cameron-makes-another-gaffe-this-election-is-all-about-my-careersorry-i-mean-country-10218341.html [Online; accessed 18-August-2020].

[5] Nicole Ellison, Rebecca Heino, and Jennifer Gibbs. 2006. Managing impressions online: Self-presentation processes in the online dating environment. Journal of computer-mediated communication 11, 2 (2006), 415-441.

[6] Shelly D. Farnham and Elizabeth F. Churchill. 2011. Faceted Identity, Faceted Lives: Social and Technical Issues with Being Yourself Online. In Proceedings of the ACM 2011 Conference on Computer Supported Cooperative Work (Hangzhou, China) (CSCW'11). Association for Computing Machinery, New York, NY, USA, 359-368. https://doi.org/10.1145/1958824.1958880

[7] GitHub. [n.d.]. Managing disruptive comments. https://docs.github.com/en/github/building-a-strong-community/managing-disruptive-comments. Accessed: 2020-09-09.

[8] Erving Goffman et al. 1978. The presentation of self in everyday life. Harmondsworth London.

[9] Russell Golman and George Loewenstein. 2015. Curiosity, information gaps, and the utility of knowledge. Information Gaps, and the Utility of Knowledge (April 16, 2015) (2015), 96-135.

[10] Shion Guha, Eric PS Baumer, and Geri K Gay. 2018. Regrets, I've had a few: When regretful experiences do (and don't) compel users to leave Facebook. In Proceedings of the 2018 ACM Conference on Supporting Groupwork. ACM, 166-177.

[11] Andreas Gutmann and Mark Warner. 2019. Fight to be forgotten: Exploring the efficacy of data erasure in popular operating systems. In Annual Privacy Forum. Springer, 45-58.

[12] P Hovey. 2010. Real-time recovery and visualization of deleted tweets. Master's thesis, University of California (2010).

[13] Hunt, Elle. 2017. I sent a compromising message to the wrong person. How will I ever recover? https://www.theguardian.com/culture/2017/apr/28/i-sent-a-compromising-message-to-the-wrong-personhow-will-i-ever-recover.

[14] Simon Jones and Eamonn O’Neill. 2011. Contextual Dynamics of Group-Based Sharing Decisions. In Proceedings of the SIGCHI Conference on Human Factors in Computing Systems (Vancouver, BC, Canada) (CHI '11). Association for Computing Machinery, New York, NY, USA, $1777-1786$. https://doi.org/10.1145/1978942.1979200

[15] Sara Kiesler, Jane Siegel, and Timothy W McGuire. 1984. Social psychological aspects of computer-mediated communication. American psychologist 39, 10 (1984), 1123.

[16] Mark L. Knapp, Laura Stafford, and John A. Daly. 1986. Regrettable Messages: Things People Wish They Hadn't Said. Journal of Communication 36, 4 (1986), 40-58. https://doi.org/10.1111/j.1460-2466.1986.tb01449.x

[17] Kuebelback, Amy. 1991. Getting the Message : E-Mail is fast and efficient. But it isn't always private-and that can mean big trouble for users. https://www.latimes.com/archives/la-xpm-1991-09-04-vw-1539-story.html [Online; accessed 18-August-2020].

[18] Myron W Lustig, Jolene Koester, and Rona Halualani. 2006. Intercultural competence: Interpersonal communication across cultures. Pearson/A and B.

[19] Nora McDonald, Sarita Schoenebeck, and Andrea Forte. 2019. Reliability and Inter-rater Reliability in Qualitative Research: Norms and Guidelines for CSCW and HCI Practice. Proceedings of the ACM on Human-Computer Interaction 3, CSCW (2019), 1-23.

[20] Margaret L Mclaughun, Michael J Cody, and H Dan O'Hair. 1983. The management of failure events: Some contextual determinants of accounting behavior. Human Communication Research 9, 3 (1983), 208-224.

[21] Sarah McRoberts, Haiwei Ma, Andrew Hall, and Svetlana Yarosh. 2017. Share first, save later: Performance of self through Snapchat stories. In Proceedings of the 2017 CHI Conference on Human Factors in Computing Systems. ACM, 6902-6911.

[22] Sandra Metts and William R Cupach. 1989. Situational influence on the use of remedial strategies in embarrassing predicaments. Communications Monographs 56, 2 (1989), 151-162.

[23] Janet R Meyer. 2011. Regretted messages: Cognitive antecedents and post hoc reflection. fournal of Language and Social Psychology 30, 4 (2011), 376-395. 
[24] Janet R Meyer and Kyra Rothenberg. 2004. Repairing regretted messages: Effects of emotional state, relationship type, and seriousness of offense. Communication Research Reports 21, 4 (2004), 348-356.

[25] Bradley M Okdie, Rosanna E Guadagno, Frank J Bernieri, Andrew L Geers, and Amber R Mclarney-Vesotski. 2011. Getting to know you: Face-to-face versus online interactions. Computers in human behavior 27, 1 (2011), 153-159.

[26] Theodor Schnitzler, Christine Utz, Florian M Farke, Christina Pöpper, and Markus Dürmuth. 2018. User Perception and Expectations on Deleting Instant Messages - or - "What Happens If I Press This Button?". In European Workshop on Usable Security (EuroUSEC).

[27] Peter Schönbach. 1980. A category system for account phases. European fournal of Social Psychology 10, 2 (1980), 195-200.

[28] Marvin B Scott and Stanford M Lyman. 1968. Accounts. American sociological review (1968), 46-62.

[29] Manya Sleeper, Justin Cranshaw, Patrick Gage Kelley, Blase Ur, Alessandro Acquisti, Lorrie Faith Cranor, and Norman Sadeh. 2013. I read my Twitter the next morning and was astonished: A conversational perspective on Twitter regrets. In Proceedings of the SIGCHI conference on human factors in computing systems. ACM, 3277-3286.

[30] Statista. 2019. Most popular messaging apps 2019. https:/www.statista.com/statistics/258749/most-popular-global-mobile-messenger-apps/ [Online; accessed 06-September-2019].

[31] Statista. 2019. U.S. WhatsApp users 2019. https://www.statista.com/statistics/558290/number-of-whatsapp-users-usa/ [Online; accessed 06-September-2019].

[32] James T Tedeschi and Marc Reiss. 1981. Verbal strategies in impression management. The psychology of ordinary explanations of social behavior 271, 309 (1981), 792-799.

[33] Catalina L Toma and Jeffrey T Hancock. 2010. Reading between the lines: linguistic cues to deception in online dating profiles. In Proceedings of the 2010 ACM conference on Computer supported cooperative work. 5-8.

[34] Catalina L Toma and Jeffrey T Hancock. 2012. What lies beneath: The linguistic traces of deception in online dating profiles. fournal of Communication 62, 1 (2012), 78-97.

[35] Jeffrey W Treem, Paul M Leonardi, and Bart van den Hooff. 2020. Computer-Mediated Communication in the Age of Communication Visibility. Fournal of Computer-Mediated Communication 25, 1 (01 2020), 44-59. https://doi.org/10.1093/jcmc/zmz024 arXiv:https://academic.oup.com/jcmc/articlepdf/25/1/44/32961193/zmz024.pdf

[36] Yang Wang, Gregory Norcie, Saranga Komanduri, Alessandro Acquisti, Pedro Giovanni Leon, and Lorrie Faith Cranor. 2011. I regretted the minute I pressed share: A qualitative study of regrets on Facebook. In Proceedings of the seventh symposium on usable privacy and security. ACM, 10.

[37] Mark Warner, Andreas Gutmann, M Angela Sasse, and Ann Blandford. 2018. Privacy Unraveling Around Explicit HIV Status Disclosure Fields in the Online Geosocial Hookup App Grindr. Proceedings of the ACM on Human-Computer Interaction 2, CSCW (2018), 181.

[38] Mark Warner, Agnieszka Kitkowska, Jo Gibbs, Juan F Maestre, and Ann Blandford. 2020. Evaluating'Prefer not to say'Around Sensitive Disclosures. In Proceedings of the 2020 CHI Conference on Human Factors in Computing Systems. 1-13.

[39] Mark Warner, Juan F Maestre, Jo Gibbs, Chia-Fang Chung, and Ann Blandford. 2019. Signal Appropriation of Explicit HIV Status Disclosure Fields in Sex-Social Apps used by Gay and Bisexual Men. In Proceedings of the 2019 CHI Conference on Human Factors in Computing Systems. ACM, 692.

[40] Mark Warner and Victoria Wang. 2019. Self-censorship in social networking sites (SNSs)-privacy concerns, privacy awareness, perceived vulnerability and information management. fournal of Information, Communication and Ethics in Society (2019).

[41] Pamela Wisniewski, Heather Lipford, and David Wilson. 2012. Fighting for my space: Coping mechanisms for SNS boundary regulation. In Proceedings of the SIGCHI Conference on Human Factors in Computing Systems. 609-618.

[42] Lu Zhou, Wenbo Wang, and Keke Chen. 2016. Tweet Properly: Analyzing Deleted Tweets to Understand and Identify Regrettable Ones. In Proceedings of the 25th International Conference on World Wide Web (Montréal, Québec, Canada) (WWW'16). International World Wide Web Conferences Steering Committee, Republic and Canton of Geneva, CHE, 603-612. https://doi.org/10.1145/2872427.2883052 